\title{
Sciendo
}

DOI: 10.1515/aon-2019-0003

\section{AIOSAT - AUTONOMOUS INDOOR \& OUTDOOR SAFETY TRACKING SYSTEM}

\author{
Iñigo Adin \\ CEIT Universidad de Navarra, Tecnun San Sebastian, Spain \\ iadin@ceit.es \\ Paul Zabalegui \\ CEIT Universidad de Navarra, Tecnun San Sebastian, Spain pzabalegui@ceit.es \\ Alejandro Perez \\ CEIT Universidad de Navarra, Tecnun San Sebastian, Spain \\ perez@ceit.es \\ Jaione Arrizabalaga \\ CEIT Universidad de Navarra, Tecnun San Sebastian, Spain jarrizabalaga@ ceit.es \\ Jon Goya \\ CEIT Universidad de Navarra, Tecnun San Sebastian, Spain \\ jgoya@ceit.es \\ Jaizki Mendizabal \\ CEIT Universidad de Navarra, Tecnun San Sebastian, Spain jmendizabal@ ceit.es
}

\begin{abstract}
Even though satellite-based positioning increases rescue workers' safety and efficiency, signal availability, reliability, and accuracy are often poor during fire operations, due to terrain formation, natural and structural obstacles or even the conditions of the operation. In central Europe, the stakeholders report a strong necessity to complement the location for mixed indoor-outdoor and GNSS blocked scenarios. As such, location information often needs to be augmented. For that, European Global Navigation Satellite System Galileo could help by improving the availability of the satellites with different features. Moreover, a multi-sensored collaborative system could also take advantage of the rescue personnel who are already involved in firefighting and complement the input data for positioning.

The Autonomous Indoor \& Outdoor Safety Tracking System (AIOSAT) is a multinational project founded through the Horizon 2020 program, with seven partners from Spain, Netherlands and Belgium. It is reaching the first year of progress (out of 3) and the overarching objective of AIOSAT system is to advance beyond the state of the art in tracking rescue workers by creating a high availability and high integrity team positioning and tracking system. On the system level approach, this goal is achieved by fusing the GNSS, EDAS/EGNOS, pedestrian dead reckoning and ultra-wide band ranging information, possibly augmented with map data. The system should be able to work both inside buildings and rural areas, which are the test cases defined by the final users involved in the consortium and the advisory board panel of the project
\end{abstract}

Keywords - GNSS, Galileo, EDAS, tracking, firefighting, multisensor, fusion, communication optimization

\section{INTRODUCTION}

Fire has historically had a large physical impact on cities and landscapes, becoming also a contributing factor to social and structural transformations. Today, Europe cities and forests remain highly susceptible to fire events, as expressed in the everyday occurrence of building fires and in the seasonal occurrence of forest 
fires with which almost everyone is familiar. Modern architecture make building fires more dangerous than they already are: for example, the tendency towards large, open buildings with high ceilings and atriums poses an increased risk to firefighters battling fire. On the other hand, Climatic Change and cultural changes in land use explain a new class of forest fires with extension and intensity magnitudes that exceed the capacity of existing suppression services.

Fires remain deadly and expensive events, both with respect to economic and human losses. Europe suffers more than 4000 deaths and 70000 hospitalizations every year due to fire and smoke related injuries. The total annual cost of fire damage in Europe is estimated in 126 billion, which is equivalent to $1 \%$ of European GDP [1].

There are more than 2.7 million firefighters across EU countries, 85 per cent of whom are volunteers [DAVIS]. Being formed by only professionals, or a mix of volunteers and professionals, all firefighters agencies have a common set of priorities when facing a fire incident, which are the in the following order:

1. Protecting of life of citizens and themselves

2. Incident stabilization through fire control

3. Protection and conservation of Property and Environment

Our first goal as firefighters is to save lives. The fire service has a long history of aggressive search and rescue operations as an initial priority of first arriving fire companies. History (and firefighter fatalities) also reflects that firefighters are exposed to the greatest risk of injury and death during primary search and rescue operations [2].

Firefighters use PPE (personal protective equipment) that allows them to operate in life-threatening conditions (e.g. breathing apparatus for toxic atmospheres) when doing offensive attacks to structural and rural fires. Standard Operating Procedures (SOP) of Fire Services commonly establish a risk-assessment methodology that governs the overall fire suppression operations and in particular safety-related aspects for fireground fighters. The golden safety rules are: i) that firefighters work together in groups of minimum two persons so that they can help each other in case of trouble; and ii) that their positions are known at all times by the incident chief, so that evacuation and/or rescue can be conducted efficiently in case of increasing risks situations or when responders are in distress. To communicate their positions, firefighters use their radio communication devices (sometimes equipped with GNSS or other kind of position reporting) and on PASS (Personal Alert Safety System) in the specific case of structural fires, which raises a loud alarm. Unfortunately, the harsh conditions occurring in many fires with zero-visibility due to smoke, high noise, collapsing structures or radical fire direction changes due to wind gusts in forest environments make that firefighters get disoriented, separated from their partners, injured and not able to communicate verbally their position due to injury or lack of coverage. In these conditions, when the incident chief notices the missing responder, it must deploy the rescue team being aware that they have usually just a few minutes to find and save the fallen responder which is in an uncertain location.

The situation exposed above, which may likely result in health and/or life losses, justifies that fire services SOPs dictate that the fire chief in charge must assess if poor radio coverage or poor/unavailable positioning means (e.g. no GNSS coverage in dense forest) exists for responders. In that case, and following the SOPs, they will take a conservative extinction approach to avoid the excessive risks and even fully cancel operations, resulting in highest fire damages.

The goal of AIOSAT project is to develop a solution that improves the safety of firefighters in the form of a GNSS and multisensorized based team-tracking system which provides reliable, real-time situational awareness to attacking firefighters and their chief command. Thus, key safety operations, namely personnel accounting, mayday alerting, rescue and evacuation can be performed quickly and effectively. 
AIOSAT will have a major primary impact in reducing firefighter deaths and health problems. By directly reducing life and health risks of fireground responders, AIOSAT will allow to increase safety trust of firefighters and fire chiefs, resulting into higher success of suppression operations and therefore into reduced fire damage.

\section{AIOSAT CONCEPT AND ARCHITECTURE}

AIOSAT aims to improve current fire responder safety when operating in environments with harsh signal propagation conditions. AIOSAT has defined the following points as the main objectives of the project:

- Enable a high availability and high integrity team positioning and tracking system based on GNSS, EGNOS and fusion of IMU, RF-ranging and map data, able to work inside buildings and rural areas.

- Develop a set of tracking and alerting application protocols for fire services which radically improve responder safety by reducing life and health risks during fire suppression operations within structures and forest environments.

- Reduce the impact of fires on health, environment and economy by enabling improved fire suppression operations founded on increased safety trust of firefighters and fire chiefs.

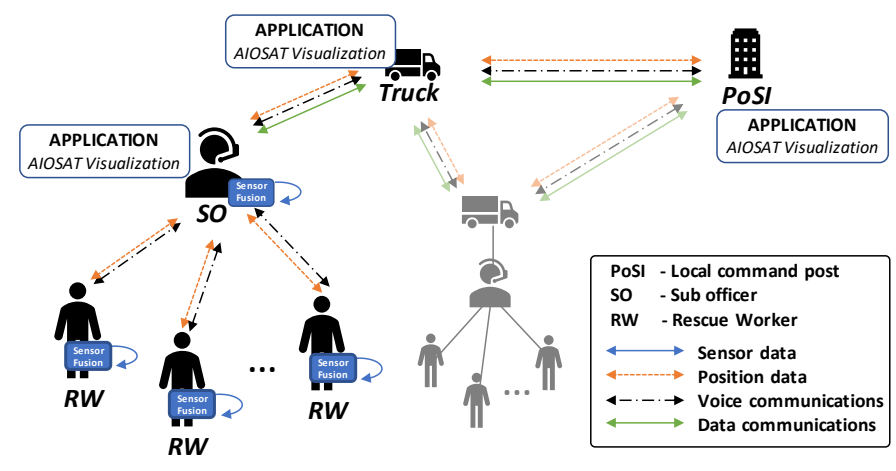

Figure 1. AIOSAT concept and proposed architecture.

Rescue teams have a hierarchical structure (see Figure 1) and in this case, the AIOSAT architecture has divided the solution in two major components having different characteristics: portable system for the rescue workers and a system for the trucks. The Tracking and Alert Application complements this.

The portable system has the following requirements:

- Continuously transmit the position of the rescue workers.

- Allow data communication of the team Sub Officer (SO) with the rescue workers and the Local Command Post at Incident (POSI) to exchange commands and alerts related to generic mission operations and in particular to prevent rescue workers from entering dangerous locations.

- Rescue workers receive the different alerts having interaction with the system, brigades and POSI taking advantage of the added value information that AIOSAT provides ensuring also the safety of their dependents.

- Receive required information from the POSI for positioning augmentation enhancing accuracy (differential correction data on the pseudo ranges) and integrity monitoring techniques, such as different Receiver Autonomous Integrity Monitoring (RAIM). 
The system deployed in the track on the other hand has the following requirements:

- Continuously receive the position of the rescue workers (see Figure 1) and track the rescue workers.

- Setup a system to retrieve real-time differential GNSS corrections. The proposed alternative is the use of EGNOS, however to increase the availability of the corrections in harsh environments where EGNOS is not available, NTRIP client (Networked Transport of RTCM via Internet Protocol), such as EDAS is also considered to obtain the differential GNSS corrections.

- Transmit the differential GNSS corrections and integrity over the AIOSAT communication system

- Transmit required orders to prevent rescue workers from entering irreversible risky locations (alarms).

- Interface with existing on-board systems and external networks to access relevant information needed for the mission, e.g. outdoor and indoor maps.

- Overall management and monitoring of the AIOSAT network and devices.

In order to succeed and meet the defined goals, the key innovation target of AIOSAT is the integration of multiple sensor data leading to a position and a Tracking and Alert intelligent system. An application backend enables the brigades and mission commanders to track the location of the rescue workers during a mission, based on map and status information from every First Responder node.

In Figure 1, the overall architecture and the data flows between the actors in the AIOSAT system are shown. Each rescue workers is equipped with a sensor pack consisting of GNSS, PDR and UWB sensor modules and an embedded platform that collects and process the sensors' data and exchanges the estimated location with other actors in a rescue team. The locations of the rescue workers are communicated through a radio link to the sub-officer, who in turn forwards it to the POSI. Both the sub-officer and the POSI have the ability to observe the locations of the rescue workers in real-time through a visualization application. The data links are realized by means of Bluetooth Low Energy (BLE), Long Range Wide Area Network (LoRaWAN) and Narrow-Band IoT (NB-IoT). The main communication channel is LoRaWan for the long range information transmission. The main drawback of this technology is the reduced bandwidth and as a result an efficient management of the communication channel has to be carried out, having as main impact the reduction of data transmission between the different actors and consequently the selection of the proper data transmission format. It is important to highlight that not all communication channels are utilized at the same time and some are meant as fall-backs only. One important point is that the voice communication channel is neither considered nor disturbed by the AIOSAT location and tracking module. This is done to increase the reliability of the alerts by having and independent communication channels as the commonly used TETRA or DRM. 


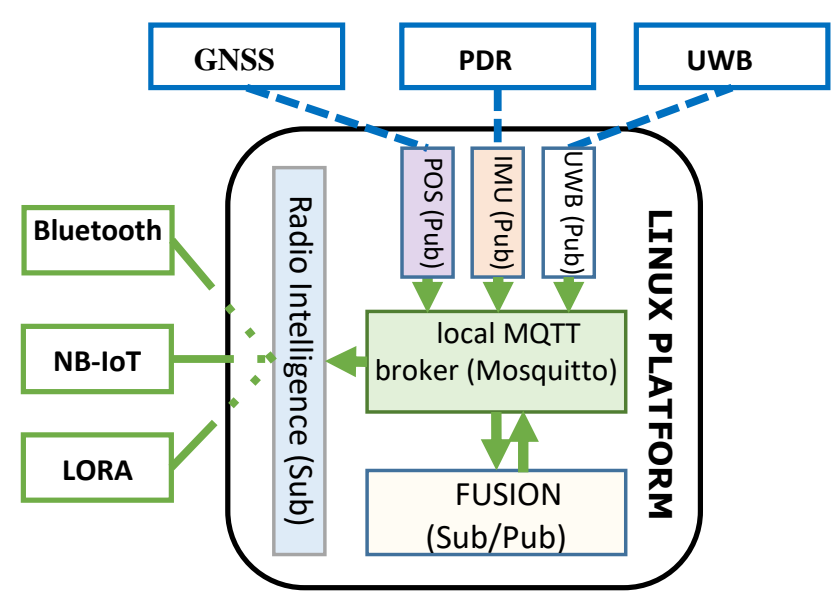

Figure 2. AIOSAT architecture.

AIOSAT proposes a publisher/subscriber architecture in order to decouple the architecture from the particular technologies and allow a faster inclusion and deployment of new technologies. Additionally, the visualization platform is independent to the system and can be used by any of the involved actor in the chain of command providing enhanced capabilities and information to support reliable decision making processes and reducing the risk of involved rescue workers to get into the fireground.

\section{FUSION ALGORITHM}

The positioning algorithm of AIOSAT systems are based on a multi-sensor approach to enhance the availability and integrity of the obtained position estimate. It is important to mention that during the project different scenarios are going to be covered for the validation of AIOSAT. On one hand the forestall fires in rural areas and on the other hand underground or mixed activities (such as parking or underground floors in building) in urban areas. Thus, as it can be foreseen the use of the multi-sensor approach is mandatory due to the different characteristic of each of the covered environments.

It is important to mention that in this section only the technologies related with the positioning are going to be covered. However, the limitations of the communication technologies have impact on how the positioning algorithms is implemented and how the data is used. The technologies that are going to be covered are the following (with the main actors from the project consortium):

- PDR based on inertial measurement units (INERTIA)

- Distance measurements between the members of a team with UWB technology (RMA)

- Augmented GNSS positioning module (CEIT)

- Sensor fusion algorithm (SAXION)

- The communication technologies are implemented by Integrasys and RMA

Even if the three technologies have their importance to obtain the position solution and to enhance the operational performance of the positioning system under the above mentioned scenarios, the GNSS and its augmentation will be covered in more detail by this paper. 


\section{A. PDR based on Inertial Measurement Units (IMU)}

The integration of IMU technology to aid GNSS positioning is widely used. This approach is well-known and commonly used in the high-tech aeronautical domain for example. However, research towards low cost solutions has been a trend during the last years. This is related with the constraint of having a stand-alone navigation grade Inertial Measurement Unit (IMU), which seemed too expensive and heavy even if it clearly has a clear advantage by being more immune to GPS outages. Solutions for which the target price cannot exceed too much due to the number of systems to be deployed on the field, such as for the proposed scenarios where several recuse workers intervene, this solution is unfeasible. Thus, the use inertial aiding and inertial coasting using low-cost micro-electromechanical (MEMS) miniature inertial measurement units are the most suitable approach for this.

Once the IMU grade is constrained by the final application, the key point is how the information obtained from the IMU is integrated with the rest of the technologies. As it is commonly known IMU increase the availability of the positioning solutions up to a $100 \%$, however the error introduced by this technology increases with the time if the error is not reseted.

For aeronautical, or other transport means, the tightly (or deeply) coupled integration approach is normally used. GNSS-INS deep integration increases application robustness, service availability, integrity, accuracy, and precision. The main advantages of GNSS-INS deep integration are:

- Acquisition and tracking of weaker signals and better quality of measurements

- Navigation solution available with fewer than four satellites in view and even under full signal blockage

- Nearly instant reacquisition after signal blockage

- Increased robustness to interference and cycle slips

However, during this project the use of loosely coupled scheme has been selected due to the aim of introducing other technologies not only GNSS and IMU and to speed-up the integration phase of the solution, as it comes from different partners. Thus, the IMU computes its own information and provides the required output to the fusion algorithms.

But this means that the error has to be reseted in some way in order to improve the capabilities of the IMU sensor and enhance also the performance.

AIOSAT included IMU technology in the shoes. These shoes can be employed by firefighters to aid in determining their location. This approach takes advantage of the zero-velocity update (ZUPT) aided inertial navigation system (INS). This reduces the location and orientation drift by resetting the error taking advantage of the zero velocity of the foot one it is stepping on the ground. The knowledge of having a zero velocity at this particular points allows the determination of the error that currently the sensor is having and isolating it at the position estimation calculation.

As it can be seen, this solution will enhance the availability of the positioning when other technologies are not available but as it is expected the performance obtained with IMU are not recommended for long time lapses without other supporting technologies. In this case, AIOSAT system incorporates inter-ranging among the firefighters involved in the intervention by means of UWB technology.

\section{B. Distance measurements between the members of a team with UWB technology}

A solution to improve the indoor positioning is to make use of UWB radio waves. The principle is to measure or estimate distances between UWB radio nodes based on the time of flight (TOF) measurements. 
Nowadays, miniaturised modules integrating a UWB IEEE802.15.4a radio that support time of flight (TOF) and time difference of arrival (TDOA) measuring schemes are commercially available on the market. It is expected that position accuracy of $50 \mathrm{~cm}$ can be achieved even in harsh indoor scenarios with multi-path propagation.

Traditionally, a realtime indoor localization systems (RTLS), based on Ultra-Wideband (UWB) Radio Technology system consist of multiple fixed reference points, called anchor nodes, with known position and mobile tags with unknown position. The tags try to measure or estimate its position relative to the anchors. The position of the tags is then calculated based on this information.

In the AIOSAT system, it would be impractical to set out anchor nodes. However, IEEE802.15.4a UWB radios can be integrated in the rescue worker portable system to measure inter-distances between neighboring rescue worker nodes. This information can be used to enhance the position estimate of rescue worker nodes with low confidence on their position, e.g. nodes with poor or no GNSS reception.

\section{Augmented GNSS positioning module}

GNSS is the angular key of AIOSAT positioning solution proposed for the rural environments and the initial position estimate for the global positioning that will support a geolocation of the rescue workers when getting into the buildings.

The aim of GNSS is not only providing the position estimate but having a reliable and accurate solution reference for the rest of technologies and vital for the rescue workers. With this aim, it is important to take advantage of the current strategies for a reliable positioning solution.

First of all, thinking on a stand-alone point of view the GNSS receiver could take advantage of multiconstellation (Galileo and GPS) and multi-frequency features (L1/E1, L2, L5/E5, etc.). As mentioned for the IMU case, one of the main issues is the cost of the different components used in AIOSAT system. The better the capabilities of the receiver and the antenna, the higher the cost of the components. Thus, a trade-off has to be reached and the use of low-cost receivers are considered in the portable systems. This means that at least Galileo E1 and GPS L1 are supported. However, thanks to the advances and the cost reduction of the receiver, newer and better low-cost receiver are expected and thus fast migration to them will be required (see Section Błąd! Nie można odnaleźć źródła odwołania.).

Even if short-term improvements of the receivers are expected, it is important to take advantage as much as possible of the current status of the technology according to the market demands. In this case, the higher-end receiver and antenna could be placed in the truck. This reduces the overall cost of the solution but with the aim of taking advantage of them. Truck are placed in relative safe and close position with high bandwidth communication links based on the operation procedures. AIOSAT aims to include other requirements such as placing the truck in a good visibility location regarding GNSS, as it will be used in several ways.

- Augmentation acquisition

- $\quad$ RTK base station

In order, to improve the positioning of the rest of the rescue workers, the truck will work as a reference point for working as a RTK bases station. The truck will have two working modes one during the dispatch of the rescue workers, having a dynamic positioning configuration that can be used for logistics and fleet control. And the second options is the most interesting one for AIOSAT with a static mode. In this static mode, the truck will act as a central point in order to receive corrections from the augmentation services NTRIP servers which send RTCM messages to correct receivers position estimations. This will allow a faster convergence time compared to precise-point-position (PPP) solutions. Additionally, it is important to take advantage of the communication link that is deployed by AIOSAT to allow relying the information based on the truck position 
acting as a RTK base station. As mentioned, the wider communication link deployed is based on LoRa, which has long rage coverage capabilities in determent of the bandwidth. This introduces a need of filtering the messages and reducing them as much as possible to avoid the communication congestion and ensuring the alerting information as it is the most critical to warn the rescue workers evacuation.

Radio Technical Commission for Maritime Services (RTCM) is the standard for the transmission of the augmentation information. In this case, AIOSAT will focus its analysis on the use of RTCM3 [3] as it cover differential GNSS information. RTCM has already worked on the optimization of the message and the information sent. For example, there are different messages defined that have a more reduced number of fields having full and reduced version of messages. In this case, the 1001, 1002 and 1004 messages are analyzed due to the fact that EDAS mountpoints usually provide the full message for the correction by means of 1004 messages and it should be converted into 1001 or 1002 messages in order to have a reduction of the data. These messages send the GPS correction observables L1. As mentioned, the rescue workers will have lowcost receiver supporting GPS L1 and thus this information will be the one selected to be transmitted.

\begin{tabular}{|l|l|l|}
\multicolumn{4}{|c|}{$\begin{array}{c}\text { Mable 1. RTCM Message Type. } \\
\text { Message } \\
\text { Type }\end{array}$} & \multicolumn{1}{|c|}{ Message Name of Bytes } \\
\hline 1001 & $\begin{array}{l}\text { L1-Only GPS RTK } \\
\text { Observables }\end{array}$ & $8.00+7.25 * \mathrm{Ns}$ \\
\hline 1002 & $\begin{array}{l}\text { Extended L1-Only GPS } \\
\text { RTK Observables }\end{array}$ & $8.00+9.25 * \mathrm{Ns}$ \\
\hline 1004 & $\begin{array}{l}\text { Extended L1\&L2 GPS RTK } \\
\text { Observables }\end{array}$ & $8.00+15.625 * \mathrm{Ns}$ \\
\hline
\end{tabular}

Table 1 shows the different amount of data needed based on the number of satellites (Ns). The first 8 bytes are linked to the header of the message and after it the different information of each of the satellites. Thus, the aim of the filtering is as the RTCM does it can follow several paths.

- Reduction of the unnecessary fields of the satellites (convert 1004 into a 1002 or 1001 message)

- Reduction of not changing field over the time (send full message periodically or on changes)

- Use of compression protocols.

Regarding the reduction of the unnecessary fields, in addition to the conversion of the message to the reduced format, other field can be suppressed if they are not used. Reaching reductions of around 53\% for 1001 and $40 \%$ for 1002 . As mentioned, also reduction of unnecessary data can be suppressed, for instance correction of satellites that the truck is not receiving and assuming that the rescue workers will not receive them neither. This solution of reduction of fields are the most straightforward, however other techniques can be also used or combined with them.

Other alternative is to send the information based on a reference message, only the non-repeated chunks of positioning data will be sent through the communication channels.

As the goal of our filtering process is to reduce the amount of data forwarded on the communication channels while keeping the real-time flow. Every RTCM3 message can be processed on the go, just the nonrepetitive data will be kept and the filtered message will be sent to the output as explained in Figure 3. 


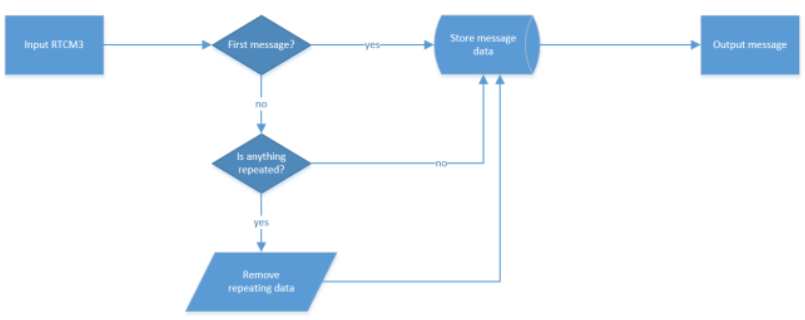

Figure 3. RTCM3 message filtering process.

At the same time, the receiving end will need to reconstruct the message in the same way as it was filtered. This means that the program will keep track of the received message chunks and fill the missing parts with the ones previously gathered. The output will be a full RTCM3 message with its corresponding fields as it was first received before the filter. The flow of the reconstruction part of this mechanism is shown in Figure 4.

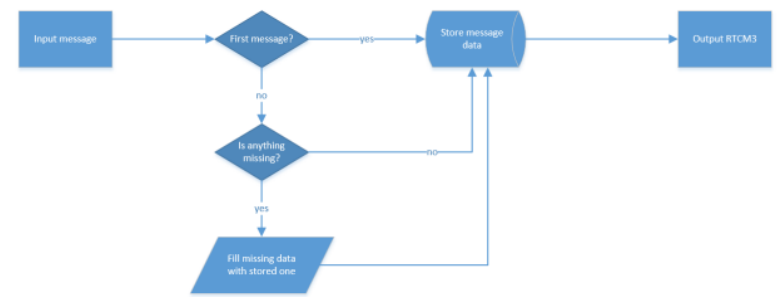

Figure 4. RTCM3 message recovering process.

RTCM transmission optimization has been already done in previous researches by implementing compression protocols, such as PECOG protocol [5], which performs data compression based on differential entropy coding. The complexity of the compression method is based on the analysis of the information flow in order to set different codification based on the entropies of the received information.

During the project the selection of simple filtering methods are applied such as the reduction of unnecessary field and transmission of the non-repetitive data.

Thanks to all these efforts, the rescue workers will receive the augmentation information into their systems to have a higher integrity solution and to take advantage of augmentation and Galileo information obtained in the truck.

\section{Sensor fusion algorithm}

The technologies and information used for the positioning algorithms are pedestrian dead-reckoning, UWB inter-distances and GNSS position. AIOSAT proposed a particle filter approach to determine the position estimate. Particle filter falls under sequential Monte Carlo methods, where lots of possible outcomes are generated (see Figure 5), from them based on the different obtained input information wrong ones between all of them are discarded (see Figure 6) and the process is iteratively repeated.

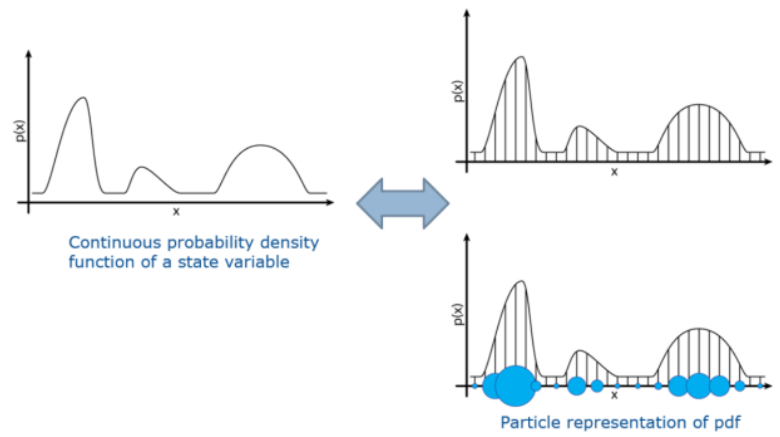

Figure 5. Particle definition based on the probability density function. 


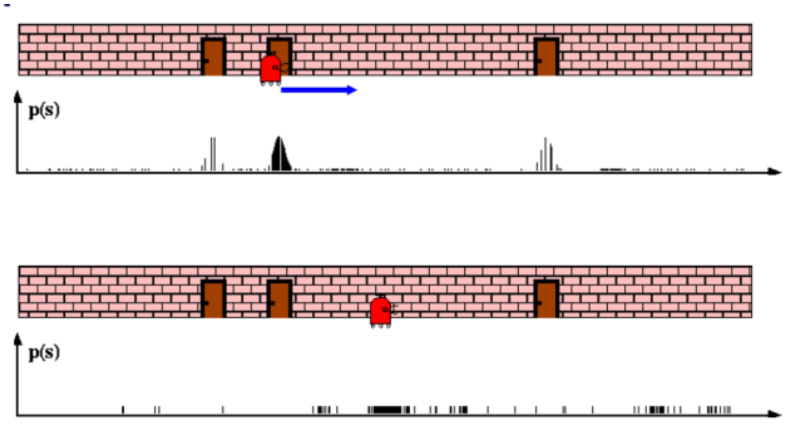

Figure 6. Example of the position probability.

One of the most important point where the complexity resides is in the strategy for the resampling and generation of the particle representation. Different alternatives are possible such as: multinominal, stratified, systematic, etc.

Once the full system is integrated, the next point is to generate the real information to test the different alternatives and determine how the solution are going to be validated in order to allow a fair comparison between different alternatives and the accomplishment of the requirements derived from the end-users.

\section{Preliminary field-test and Validation}

During the first integration, first field-test were carried out in order to test the integration and gather real data for the further development of the sensor fusion solution and resampling method that fits better for AIOSAT. This is a necessary step due to the need of synchronized information of all the technologies and sensors involved in the systems. The preliminary analysis of the data obtained clearly shows the need of including correction in order to enhance the performance of the obtained position estimate (see Figure 7 and

Figure 8). This means that the path followed by the project seems to be correct, as it can be seen the results are obtained for outdoor scenario, this means that further field-test will have to be carried out to confirm the preliminary analysis and extend the field-test to both proposed scenarios.

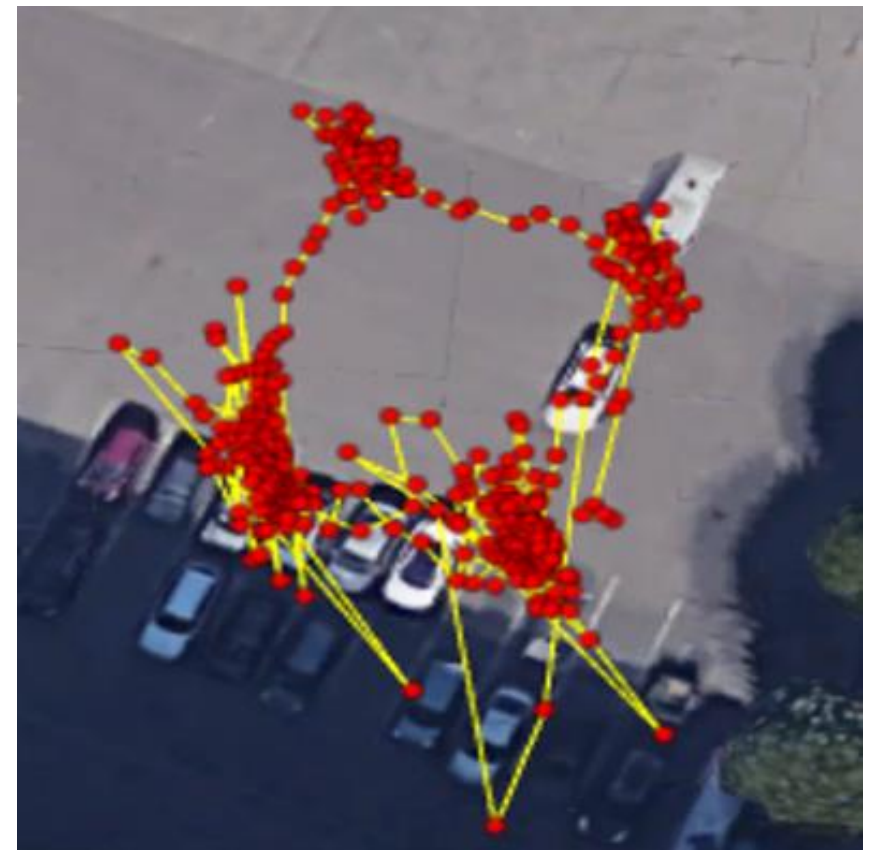

Figure 7. Field-test results without correction. 


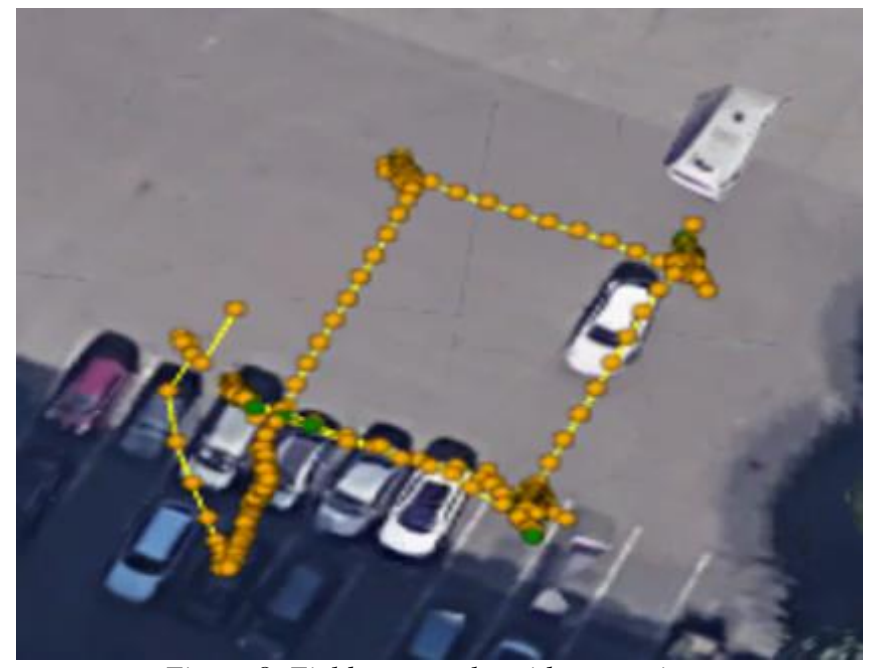

Figure 8. Field-test results with corrections.

Apart from the field-test one of the key point is the validation process in order to ensure a common framework to fairly compare the different variants. In this case, AIOSAT will be validated by following the ISO 18305 [9]. This standard defines different procedures for the validation of real time location services for different areas such as emergency services. It defines the appropriate performance metrics and result visualization for the proper evaluation of this kind of location and tracking services. Following this standard will ensure the proper evaluation of the AIOSAT system having a fair evaluation of the requirements.

\section{CONCLUSIONS AND FUTURE STEPS}

The AIOSAT project aims at providing high-reliability location information during rescue operations. Its design focuses on modularity and ensuring data availability by integrating multiple sensors such as GNSS, IMU and UWB ranging subsystem, from which information, a fusing algorithm obtains the localization data. The communication technologies are various, starting with BLE and LORA but extendable to others such as NB-IoT or LTE. In the coming months, the partners will work towards the integration of all the subcomponents. Moreover, the field tests, simulating real-life scenarios will be carried out to help collecting sensor data and evaluate the fusion algorithm.

\section{ACKNOWLEDGMENT}

Results incorporated in this paper received funding from the European GNSS Agency under the European Union's Horizon 2020 research and innovation program under grant agreement No 776425

\section{REFERENCES}

[1] White paper of Fire Safe Europe launched on 2 December 2014 in the European Parliament http://firesafeeurope.eu/wp-content/uploads/2015/06/Europe-is-playing-with-fire-_-WhitePaper_FSEU_03_12_2014.pdf

[2] Rules of Engagement for Structural Firefighting, 2012 International Association of Fire Chiefs

[3] RTCM, "RTCM STANDARD 10403.3 DIFFERENTIAL GNSS (GLOBAL NAVIGATION SATELLITE SYSTEMS) SERVICES - VERSION 3,” 2016.

[4] N.N. Brushlinsky, M. Arhens, S.V. Sokolov, P. Wagner, World Fire Statistics v.28 2018

[5] Autonomous Indoor/Outdoor Safety Tracking System (AIOSAT) webpage, http://www.aiosat.eu/

[6] J. Laarhuis, MoSeS ondersteunt brandweer, NIDV Magazine, 1-2018 
[7] M. Acton, Data-Oriented Design and C++, CppCon 2014

[8] William J. Kellar, Miles P. Moody, Transmission of RTK Corrections and Measurements using Optimal Coding, Journal of Global Positioning Systems 2006

[9] IEC, "ISO 18305 -Information technology - Real Time Locating Systems - Test and evaluation,", 2016. 